\title{
SHORT COMMUNICATION \\ Association of the AFF3 gene and IL2/IL21 gene region with juvenile idiopathic arthritis
}

\author{
A Hinks ${ }^{1}$, S Eyre ${ }^{1}$, X Ke ${ }^{1}$, A Barton ${ }^{1}$, P Martin ${ }^{1}$, E Flynn $^{1}$, J Packham², J Worthington ${ }^{1}$, Childhood \\ Arthritis Prospective Study (CAPS), UKRAG Consortium, BSPAR Study Group ${ }^{3}$ and W Thomson ${ }^{1}$ \\ ${ }^{1}$ arc-EU, Stopford Building, The University of Manchester, Manchester, UK and ${ }^{2}$ Haywood Hospital, University Hospital of North \\ Staffordshire, Stoke on Trent, Staffordshire, UK
}

\begin{abstract}
Recent genetic studies have led to identification of numerous loci that are associated with susceptibility to autoimmune diseases. The strategy of using information from these studies has facilitated the identification of novel juvenile idiopathic arthritis (JIA) susceptibility loci, specifically, PTPN22 and IL2RA. Several novel autoimmune susceptibility loci have recently been identified, and we hypothesise that single-nucleotide polymorphisms (SNPs) within these genes may also be JIA susceptibility loci. Five SNPS within the genes AFF3, IL2/IL21, IL7R, CTLA4 and CD226, previously associated with multiple autoimmune diseases were genotyped, in a large data set of Caucasian JIA patients and controls, and tested for association with JIA. We identified two susceptibility loci for JIA, AFF3 and the IL2/IL21 region and additional weak evidence supporting an association with the CTLA4 and IL7R genes, which warrant further investigation. All results require validation in independent JIA data sets. Further characterisation of the specific causal variants will be required before functional studies can be performed.
\end{abstract}

Genes and Immunity (2010) 11, 194-198; doi:10.1038/gene.2009.105; published online 14 January 2010

Keywords: autoimmune; juvenile idiopathic arthritis; CTLA4; AFF3; IL2

\section{Introduction}

Autoimmune diseases are caused by dysregulation of the immune system leading to an immune response to selftissue. Autoimmune diseases are complex genetic diseases and in the last few years great progress has been made in the search for susceptibility loci. ${ }^{1,2}$ As more confirmed autoimmune disease susceptibility loci are identified, an interesting story is emerging in that many of these loci predispose to more than one autoimmune disease. This confirms the hypothesis that shared alleles contribute to a spectrum of diseases and suggests that common immunological pathways are involved in susceptibility to these phenotypically distinct diseases. ${ }^{3}$

Juvenile idiopathic arthritis (JIA) is another complex genetic autoimmune disease characterised by chronic inflammatory disease in children. It is a group of heterogeneous disorders but encompasses all forms of arthritis of unknown aetiology that starts before the age of 16 and which persists for at least 6 weeks. ${ }^{4}$ The strategy of using information from autoimmune disease genome-wide association studies or candidate gene studies have facilitated the search for novel JIA suscept-

Correspondence: Dr A Hinks, Arthritis Research Campaign (arc)Epidemiology Unit, University of Manchester, Stopford building, Manchester M13 9PT, UK.

E-mail: Anne.Hinks@manchester.ac.uk

${ }^{3}$ Members of CAPS, the UKRAG Consortium and BSPAR Study Group are listed in the Appendix.

Received 8 April 2009; revised 15 October 2009; accepted 19 October 2009; published online 14 January 2010 ibility loci. Indeed two recently identified confirmed JIA susceptibility loci, PTPN $22^{5}$ and IL $2 R A^{6}$ are putative autoimmune susceptibility genes as they also show association with rheumatoid arthritis (RA), , , type I diabetes (T1D) ${ }^{7,8}$ and Graves' disease. ${ }^{9,10}$ In addition, using a strategy of examining confirmed RA susceptibility loci in JIA, we have recently reported evidence for association of two further loci (TRAF1/C5 and STAT4) with JIA susceptibility. ${ }^{11}$

Several novel putative autoimmune susceptibility loci have recently been identified with association with multiple autoimmune diseases. These include the IL2/21 region on chromosome $4 \mathrm{q} 23^{12,13}$ and the genes encoding IL7R, ${ }^{14-16} C T L A 4,{ }^{17} A F F 3^{14}$ and $C D 226 .{ }^{18}$ We hypothesise that these genes may also confer susceptibility to JIA and, therefore, the aim of this study was to determine whether single-nucleotide polymorphisms (SNPs) within these genes are also associated with susceptibility to JIA.

\section{Results and discussion}

In this study, using a strategy of examining previously associated autoimmune loci in JIA, we have identified association of two loci with JIA susceptibility (Table 1). First, we show association of a SNP (rs1160542) in the $5^{\prime}$ region of the AFF3 gene (Table 1), a gene that is preferentially expressed in lymphoid cells and has a potential regulatory role in lymphoid development. ${ }^{19}$ This SNP has been associated with $\mathrm{RA}^{20}$ and a perfect proxy SNP $\left(r^{2}=1\right)$, rs9653442 has been associated with 
$\mathrm{T}_{1 \mathrm{D}}{ }^{14}$ with similar odds ratio and allele frequencies to that observed in this study of JIA.

Second, we found strong association of a SNP (rs6822844) mapping within the IL2-IL21 locus on chromosome 4q27, which has previously been associated with RA, T1D and coeliac disease (Table 1). ${ }^{12,13}$ The SNP lies approximately $24 \mathrm{~kb} \mathrm{5}^{\prime}$ of the IL21 gene. The SNP lies within a block of high linkage disequilibrium, which contains four genes, KIAA1109, TENR, IL2 and IL21. As for the other diseases, it is the common allele, which predisposes to JIA. This association confirms a recently published association of the IL2_IL2 region with JIA susceptibility. ${ }^{21}$ We performed a meta-analysis of the two studies, which yielded highly significant evidence for association (odds ratio $0.7795 \%$ confidence interval 0.69 $0.87, P=1 \times 10^{-5}$ ) with no evidence for heterogeneity between the two cohorts $(P=0.81)$. This finding is interesting in light of the previous confirmed association of the IL2RA gene with JIA ${ }^{6}$ and may suggest that the IL2 pathway is particularly important in JIA susceptibility.

We found a weak trend toward association of a SNP in the IL7R gene with JIA (Table 1), in line with the previous association of this SNP with RA, T1D ${ }^{14}$ and multiple sclerosis $^{15,16}$ the common allele of the SNP was increased in cases compared with controls, although this did not achieve statistical significance. However, this study was under-powered with only $18 \%$ power to detect an effect (Supplementary Table 2). Therefore, additional independent studies and meta-analyses of this SNP will be required to confirm it as associated with JIA susceptibility. The SNP is a non-synonymous SNP within exon 6 of $I L 7 R$ and has a functional effect on gene expression, resulting in altered ratios of soluble and membranebound isoforms of the protein. ${ }^{15}$

SNPs within the CTLA4 gene, previously associated with T1D and autoimmune thyroid disease ${ }^{17}$ have previously been examined in JIA with conflicting results. ${ }^{22,23}$ This may reflect true genetic heterogeneity at this locus or may be due to the modest sample sizes used in previous investigations. We found a weak association of the CTLA4 CT60 SNP (rs3087243) with UK JIA cases (Table 1), although this study only had 53\% power to detect an effect (Supplementary Table 2). However, no evidence for association of this SNP with JIA was detected in a recent large study of US JIA families and controls. ${ }^{23}$ We used the Cochran-MantelHaenszel test to perform a meta-analysis combining data from this study and the Prahalad study; this yielded weak but statistically significant evidence for association (odds ratio $0.9295 \%$ confidence interval 0.84-1.0, $P=0.05$ ) with no evidence for heterogeneity (by Breslow-Day test) between the two cohorts $(P=0.44)$. Further analysis of this SNP in independent data sets followed by meta-analysis will be essential to robustly determine whether CTLA4 represents a JIA susceptibility locus. It is obviously a good candidate as an autoimmune susceptibility locus because of its role as a negative regulator of T-cell activation. ${ }^{17}$ Furthermore, the CT60 SNP is found within the $3^{\prime}$ untranslated region, in which the $G$ allele is associated with susceptibility to several autoimmune diseases and also has a functional effect of lower mRNA levels of the soluble CTLA4 isoform. ${ }^{17}$

Finally, a non-synonymous SNP, rs763361, in exon 7 of the CD226 gene has recently been associated with multiple autoimmune diseases including T1D, multiple 


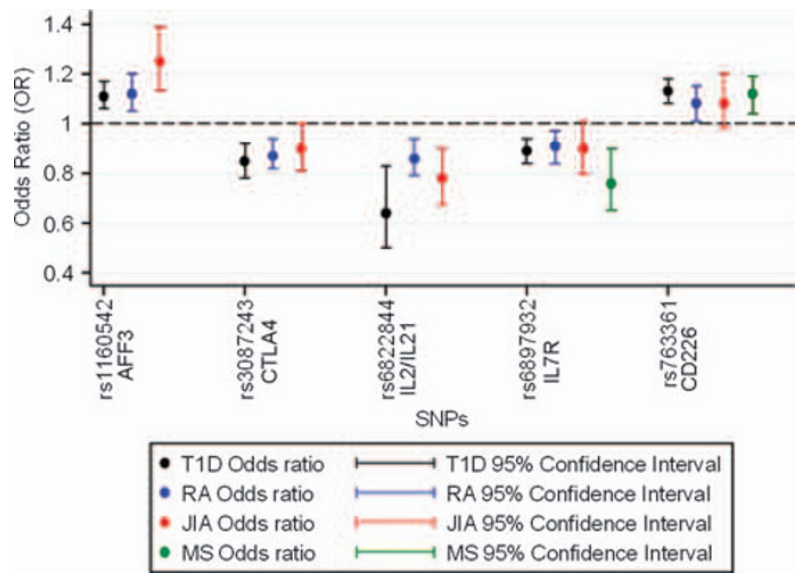

Figure 1 Plot of odds ratios for minor allele for SNPs previously associated with autoimmune disease, comparison with JIA. Plots of odds ratios and 95\% confidence intervals for the association analysis of all SNPs, results in T1D (black dots and lines), in RA (blue dots and lines), in JIA (red dots and lines) and in MS (green dots and lines). References ${ }^{12,14,16-18}$ and Barton (2009) submitted.

sclerosis and possibly autoimmune thyroid disease and RA. ${ }^{18}$ In our total JIA analysis, we found no significant association of the SNP with JIA (Table 1). However, we only had $24 \%$ power to detect an effect (Supplementary Table 2).

Figure 1 shows a comparison between the association analysis results in T1D, RA, multiple sclerosis and JIA. For all the SNPs tested, the same allele was associated with JIA as was associated with the other autoimmune diseases and effect sizes are similar. Hence, the failure to confirm association with CTLA4 and IL7R at the corrected threshold could be due to a lack of statistical power (53 and 18\%, respectively) (Supplementary Table 2). It has not always been the case for the overlapping autoimmune disease susceptibility loci, that the same allele is associated. For example in PTPN22, the minor allele of the R620W SNP is associated with greater risk of developing RA, JIA, T1D and SLE but is protective for Crohn's disease. ${ }^{24,25}$ There is also emerging data suggesting that one of the associated SNPs at the IL2RA locus confers differing risk and protective effects for T1D and multiple sclerosis. ${ }^{26,27}$

JIA is a phenotypically heterogeneous disease and can be classified into more clinically homogeneous diseases using the ILAR classification criteria (Supplementary Table 1). ${ }^{28}$ However, comparing each of the ILAR subtypes separately against controls would result in a large number of hypothesis tests. Therefore, we first examined whether there was evidence of a difference in allele frequencies between the seven ILAR subtypes. Differences between subtypes were assessed using $\chi^{2}$ tests on the $7 \times 2$ tables. Only when a difference was found $(P<0.05)$ were separate odds ratios and $95 \%$ confidence intervals calculated for the subgroups. In all cases, this was not significant $(P>0.05)$ (data not shown). Therefore, further stratification by ILAR subtype was not performed. Larger sample sizes will be required to fully examine subgroup differences.

In conclusion, adopting the strategy of targeting loci with previous evidence for association in multiple autoimmune diseases has identified two novel JIA loci, AFF3 and the IL2/IL21 locus.

\section{Conflict of interest}

The authors declare no conflict of interest.

\section{Acknowledgements}

This work was supported by the Arthritis Research Campaign: arc grant reference no: 17552. We thank David Strachan for facilitating access to the 1958 birth cohort. We acknowledge use of genotype data from the British 1958 Birth Cohort DNA collection, funded by the Medical Research Council grant G0000934 and the Wellcome Trust grant 068545/Z/02.

\section{References}

1 Lettre G, Rioux JD. Autoimmune diseases: insights from genome-wide association studies. Hum Mol Genet 2008; 17 (R2): R116-R121.

2 Zhernakova A, van Diemen CC, Wijmenga C. Detecting shared pathogenesis from the shared genetics of immunerelated diseases. Nat Rev Genet 2009; 10: 43-55.

3 Vyse TJ, Todd JA. Genetic analysis of autoimmune disease. Cell 1996; 85: 311-318.

4 Ravelli A, Martini A. Juvenile idiopathic arthritis. Lancet 2007; 369: 767-778.

5 Hinks A, Worthington J, Thomson W. The association of PTPN22 with rheumatoid arthritis and juvenile idiopathic arthritis. Rheumatology (Oxford) 2006; 45: 365-368.

6 Hinks A, Ke X, Barton A, Eyre S, Bowes J, Worthington J et al. Association of the IL2RA/CD25 gene with juvenile idiopathic arthritis. Arthritis Rheum 2009; 60: 251-257.

7 The Wellcome Trust Case Control Consortium. Genome-wide association study of 14000 cases of seven common diseases and 3000 shared controls. Nature 2007; 447: 661-678.

8 Lowe CE, Cooper JD, Brusko T, Walker NM, Smyth DJ, Bailey $\mathrm{R}$ et al. Large-scale genetic fine mapping and genotypephenotype associations implicate polymorphism in the IL2RA region in type 1 diabetes. Nat Genet 2007; 39: 1074-1082.

9 Brand OJ, Lowe CE, Heward JM, Franklyn JA, Cooper JD, Todd JA et al. Association of the interleukin-2 receptor alpha (IL-2Ralpha)/CD25 gene region with Graves' disease using a multilocus test and tag SNPs. Clin Endocrinol (Oxf) 2007; 66: 508-512.

10 Smyth D, Cooper JD, Collins JE, Heward JM, Franklyn JA, Howson JM et al. Replication of an association between the lymphoid tyrosine phosphatase locus (LYP/PTPN22) with type 1 diabetes, and evidence for its role as a general autoimmunity locus. Diabetes 2004; 53: 3020-3023.

11 Hinks A, Eyre S, Ke X, Barton A, Martin P, Flynn E et al. Overlap of disease susceptibility loci for rheumatoid arthritis (RA) and juvenile idiopathic arthritis (JIA). Ann Rheum Dis 2009; e-pub ahead of print 11 August 2009.

12 van Heel DA, Franke L, Hunt KA, Gwilliam R, Zhernakova A, Inouye $\mathrm{M}$ et al. A genome-wide association study for celiac disease identifies risk variants in the region harboring IL2 and IL21. Nat Genet 2007; 39: 827-829.

13 Zhernakova A, Alizadeh BZ, Bevova M, van Leeuwen MA, Coenen MJ, Franke B et al. Novel association in chromosome $4 q 27$ region with rheumatoid arthritis and confirmation of type 1 diabetes point to a general risk locus for autoimmune diseases. Am J Hum Genet 2007; 81: 1284-1288.

14 Todd JA, Walker NM, Cooper JD, Smyth DJ, Downes K, Plagnol V et al. Robust associations of four new chromosome regions from genome-wide analyses of type 1 diabetes. Nat Genet 2007; 39: 857-864.

15 Gregory SG, Schmidt S, Seth P, Oksenberg JR, Hart J, Prokop A et al. Interleukin 7 receptor alpha chain (IL7R) shows allelic 
and functional association with multiple sclerosis. Nat Genet 2007; 39: 1083-1091.

16 Lundmark F, Duvefelt K, Iacobaeus E, Kockum I, Wallstrom E, Khademi $\mathrm{M}$ et al. Variation in interleukin 7 receptor alpha chain (IL7R) influences risk of multiple sclerosis. Nat Genet 2007; 39: 1108-1113.

17 Ueda H, Howson JM, Esposito L, Heward J, Snook H, Chamberlain $\mathrm{G}$ et al. Association of the T-cell regulatory gene CTLA4 with susceptibility to autoimmune disease. Nature 2003; 423: 506-511.

18 Hafler JP, Maier LM, Cooper JD, Plagnol V, Hinks A, Simmonds MJ et al. CD226 Gly307Ser association with multiple autoimmune diseases. Genes Immun 2008; 10: 5-10.

19 Ma C, Staudt LM. LAF-4 encodes a lymphoid nuclear protein with transactivation potential that is homologous to AF-4, the gene fused to MLL in $t(4 ; 11)$ leukemias. Blood 1996; 87: 734-745.

20 Barton A, Eyre S, Ke X, Hinks A, Bowes J, Flynn E et al. Identification of AF4/FMR2 family, member 3 (AFF3) as a novel rheumatoid arthritis susceptibility locus and confirmation of two further pan-autoimmune susceptibility genes. Hum Mol Genet 2009; 18: 2518-2522.

21 Albers HM, Kurreeman FA, Stoeken-Rijsbergen G, Brinkman DM, Kamphuis SS, Van Rossum MA et al. Association of the autoimmunity locus 4q27 with juvenile idiopathic arthritis. Arthritis Rheum 2009; 60: 901-904.

22 Suppiah V, O'doherty C, Heggarty S, Patterson CC, Rooney M, Vandenbroeck K. The CTLA4+49A/G and CT60 polymorphisms and chronic inflammatory arthropathies in Northern Ireland. Exp Mol Pathol 2006; 80: 141-146.

23 Prahalad S, Bohnsack JF, Whiting A, Clifford B, Jorde LB, Guthery SL et al. Lack of association of functional CTLA4 polymorphisms with juvenile idiopathic arthritis. Arthritis Rheum 2008; 58: 2147-2152.

24 Vang T, Congia M, Macis MD, Musumeci L, Orru V, Zavattari $\mathrm{P}$ et al. Autoimmune-associated lymphoid tyrosine phosphatase is a gain-of-function variant. Nat Genet 2005; 37: 1317-1319.

\section{Appendix}

Childhood Arthritis Prospective Study (CAPS): Arc Epidemiology Unit, University of Manchester-Kimme Hyrich, Mark Lay, Sham Lal, Paul Gilbert, Peter Ward; Alderhey-Eileen Baildam, Carol Lydon, Lynsey Brown; Glasgow-Joyce Davidson, Janet Gardner-Medwin, Vicki Price, Jane Sim, Maureen Todd; Great Ormond StreetLucy Wedderburn, Alexandra Meijer, Julie Jones; Newcastle-Helen Foster, Mark Friswell, Michael Eltringham; Manchester-Alice Chieng, Joanne Buckley; OtherTauny Southwood

UKRAG Consortium: University of Manchester ${ }^{1}$ : Stephen Eyre, Anne Hinks, Laura J Gibbons, John Bowes, Edward Flynn, Paul Martin, Xiayi Ke, Rachelle Donn, Wendy Thomson, Anne Barton, Jane Worthington

University of Leeds's: YEAR Consortium $\dagger$, Stephen Martin, James I Robinson, Ann W Morgan, Paul Emery University of Sheffield ${ }^{3}$ : Anthony G Wilson

University of London ${ }^{4}$ : Sophia Steer

University of Aberdeen ${ }^{5}$ : Lynne Hocking, David M Reid

University of Oxford': Pille Harrison, Paul Wordsworth

${ }^{1}$ arc-Epidemiology Unit, Stopford Building, The University of Manchester, Manchester, UK
25 Barrett JC, Hansoul S, Nicolae DL, Cho JH, Duerr RH, Rioux JD et al. Genome-wide association defines more than 30 distinct susceptibility loci for Crohn's disease. Nat Genet 2008; 40: 955-962.

26 Maier LM, Lowe CE, Cooper J, Downes K, Anderson DE, Severson C et al. IL2RA genetic heterogeneity in multiple sclerosis and type 1 diabetes susceptibility and soluble interleukin-2 receptor production. PLoS Genet 2009; 5: e1000322.

27 Alcina A, Fedetz M, Ndagire D, Fernandez O, Leyva L, Guerrero $\mathrm{M}$ et al. IL2RA/CD25 gene polymorphisms: uneven association with multiple sclerosis (MS) and type 1 diabetes (T1D). PLoS ONE 2009; 4: e4137.

28 Petty RE, Southwood TR, Manners P, Baum J, Glass DN, Goldenberg $\mathrm{J}$ et al. International league of associations for rheumatology classification of juvenile idiopathic arthritis: second revision, Edmonton, 2001. J Rheumatol 2004; 31: 390-392.

29 Packham JC, Hall MA. Long-term follow-up of 246 adults with juvenile idiopathic arthritis: functional outcome. Rheumatology (Oxford) 2002; 41: 1428-1435.

30 Adib N, Hyrich K, Thornton J, Lunt M, Davidson J, GardnerMedwin J et al. Association between duration of symptoms and severity of disease at first presentation to paediatric rheumatology: results from the childhood arthritis prospective study. Rheumatology (Oxford) 2008; 47: 991-995.

31 Thomson W, Barton A, Ke X, Eyre S, Hinks A, Bowes J et al. Rheumatoid arthritis association at 6q23. Nat Genet 2007; 39: 1431-1433.

32 Purcell S, Neale B, Todd-Brown K, Thomas L, Ferreira MA, Bender D et al. PLINK: a tool set for whole-genome association and population-based linkage analyses. Am J Hum Genet 2007; 81: 559-575.

(c) This work is licensed under the Creative Commons Attribution-NonCommercial-No Derivative Works 3.0 Licence. To view a copy of this licence, visit http://creativecommons.org/licenses/by-nc$\mathrm{nd} / 3.0 /$

${ }^{2}$ Leeds Institute of Molecular Medicine, Section of Musculoskeletal Disease, University of Leeds, Leeds LS9 7TF, UK

${ }^{3}$ School of Medicine \& Biomedical Sciences, The University of Sheffield, Sheffield S10 2JF, UK

${ }^{4}$ Clinical and Academic Rheumatology, Kings College Hospital NHS Foundation Trust, Denmark Hill, London SE5 9RS, UK

${ }^{5}$ Bone Research Group, Department of Medicine \& Therapeutics, University of Aberdeen, Aberdeen AB25 2ZD, UK

'University of Oxford Institute of Musculoskeletal Sciences, Botnar Research Centre, Oxford OX3 7LD, UK YEAR Consortium: Management Team-Professor Paul Emery ${ }^{1}$, Professor Philip Conaghan ${ }^{1}$, Dr Mark Quinn ${ }^{2}$, Dr Ann W Morgan ${ }^{1}$, Dr Anne-Maree Keenan ${ }^{1}$, Dr Elizabeth Hensor $^{1}$, Julie Kitcheman ${ }^{1}$. Consultants-Dr Andrew Gough $^{3}$, Dr Michael Green ${ }^{2,3}$, Dr Richard Reece ${ }^{4}$, Dr Lesley Hordon', Dr Philip Helliwell16, Dr Richard Melsom $^{6}$, Dr Sheelagh Doherty ${ }^{7}$, Dr Ade Adebajo ${ }^{8}$, Dr Andrew Harvey ${ }^{9}$, Dr Steve Jarrett ${ }^{9}$, Dr Gareth Huson ${ }^{1}$, Dr Amanda Isdale ${ }^{2}$, Dr Mike Martin ${ }^{1}$, Dr Zunaid Karim', Prof Dennis McGonag1e ${ }^{10}$, Dr Colin Pease ${ }^{1}$, Dr Sally Cox $^{1}$. SpRs-Dr Victoria Bejarano ${ }^{1}$, Dr Jackie Nam ${ }^{1}$. Nurses-Claire Brown ${ }^{1}$, Christine Thomas ${ }^{1}$, David Pickles $^{1}$, Alison Hammond ${ }^{1}$, Beverley Neville ${ }^{3}$, Alan 
Fairclough ${ }^{4}$, Caroline Nunns, ${ }^{4}$ Anne Gill², Julie Green ${ }^{2}$, Belinda Rhys-Evans ${ }^{1}$, Barbara Padwell ${ }^{1}$, Julie Madden ${ }^{10}$, Lynda Taylor ${ }^{10}$, Sally Smith ${ }^{1}$, Heather King ${ }^{1}$, Jill Firth ${ }^{6}$, Jayne Heard7, Linda Sigsworth ${ }^{6}$. Lab Staff-Diane Corscadden $^{1}$, Karen Henshaw ${ }^{1}$, Lubna-Haroon Rashid ${ }^{1}$, Stephen G Martin ${ }^{1}$, James I Robinson ${ }^{1}$

${ }^{1}$ Section of Musculoskeletal Disease, LIMM, Leeds, UK

${ }^{2}$ York District Hospital, York, UK

${ }^{3}$ Harrogate District Hospital, Harrogate, UK

${ }^{4}$ Huddersfield Royal Infirmary, Huddersfield, UK

${ }^{5}$ Dewsbury District and General Hospital, Dewsbury, UK

'St Luke's Hospital, Bradford, UK
${ }^{7}$ Hull Royal Infirmary, Hull, UK

${ }^{8}$ Barnsley District General Hospital, Barnsley, UK

${ }^{9}$ Pinderfields General Hospital, Wakefield, UK

${ }^{10}$ Calderdale Royal Hospital, Halifax, UK

British Society of Paediatric and Adolescent Rheumatology (BSPAR) study group: M Abinum, MD, M Becker, MD, A Bell, MD, A Craft, MD, E Crawley, MD, J David, MD, H Foster, MD, J Gardener-Medwin, MD, J Griffin, MD, A Hall, MD, M Hall, MD, A Herrick, MD, P Hollingworth, MD, L Holt, MD, S Jones, MD, G Pountain, MD, C Ryder, MD, T Southwood, MD, I Stewart, MD, H Venning, L Wedderburn, MD, P Woo, MD and S Wyatt, MD.

Supplementary Information accompanies the paper on Genes and Immunity website (http://www.nature.com/gene) 